\title{
A Novel Multiple Watermark With ROI Recovery
}

\author{
Dan $\mathrm{Wu}^{*}$, Ruxing $\mathrm{Xu}$ and Jiao $\mathrm{Wu}$ \\ Department of Mathematics, China Jiliang University Hangzhou, China \\ *Corresponding author
}

\begin{abstract}
A novel multiple watermarking scheme was proposed, which could embed the reversible watermark in ROI (region of interest) by improving the Ni's algorithm, and the robust watermark in non-ROI by quantizing the low frequency coefficients. The watermarks can simultaneously achieve copyright protection and content authentication, and the ROI of the watermarked image can be recovered completely if it isn't attacked. At the same time, the multipurpose watermarks are extracted in a blind manner without requiring the original image. The experimental results illustrate that the pure payload of the proposed reversible watermarking algorithm is higher 10 percent than Ni's scheme, and the robust watermark can endure common image attacks.
\end{abstract}

Keywords-multiple watermark; reversible watermark; histogram shifting; quantization

\section{INTRODUCTION}

Copyright marking has recently become an important technique for multimedia information hiding. Its application is broad, including ownership protection[1-2], content authentication[3], side information conveyance [4] and so on.

There are many watermarking algorithms which focus on multiple robust watermarking for ownership embedding[5-8]. However, only few suggestions focus on embedding actually different watermarks. Lu et al.[3] proposed a multipurpose watermarking scheme, in which robust and fragile watermarks were simultaneously embedded for copyright protection and content authentication by quantizing a host image's wavelet coefficients. But all these algorithms can't avoid the mutual interference between different watermarks.

In this paper, we propose a novel multiple watermarking scheme which embeds the reversible watermark in ROI (region of interest) by improving the Ni's algorithm[9], and the robust watermark in non-ROI by quantizing the low frequency coefficients. The watermarks can simultaneously achieve copyright protection and content authentication, and the ROI of the watermarked image can be recovered completely if it isn't attacked. At the same time, the multipurpose watermarks are extracted in a blind manner without requiring the original image. The experimental results illustrate that the pure payload of the proposed reversible watermarking algorithm is higher 10 percent than Ni's scheme, and the robust watermark can endure common image attacks.

\section{PROPOSED MULTIPLE WATERMARKING ALGORITHM}

This section will elaborate on the proposed approach in detail. In Section A, a novel reversible watermarking algorithm is proposed to embedded the watermark in the ROI. In Section $B$ we modulate the low frequency coefficients to embed the robust watermark in the remaining image.

The ROI(region of interesting), called $I_{0}$, is the important part of an image $I(L \times L)$ and its size is $M \times N$. The reversible visible watermark (W1) is embedded in $I_{0}$, and the robust watermark (W2)is embedded in the remaining part ( $\left.{ }^{I_{1}}\right)$.

\section{A. Reversible Watermarking}

$\mathrm{Ni}$ proposed a simple reversible watermarking algorithm based on histogram shifting. We improve the algorithm, which can embed two data into three pixels using the template.

The embedding procedure includes the following steps:

S1) Generate the image's histogram $\mathrm{H}(\mathrm{x})$;

S2) In the histogram $H(x)$ find the maximum point $h(a)$ and the minimum point $h(b)$.

S3) Without loss of generality , assume $a<b$. all the pixel gray values (satisfying $\mathrm{a}<\mathrm{x}<\mathrm{b}$ ) are added by 1 ,and leave $\mathrm{a}+1(\mathrm{M})$ empty.

S4) Scan the image I, record the pixel a(L) and $a+2(R)$. According to the Template (Table1), every two data are embedded into three pixels.

TABLE I. THE RELATIONSHIP BETWEEN THE TWO MARKS AND THE THREE PIXELS

\begin{tabular}{|l|l|l|l|l|}
\hline & & & & \\
& & & & \\
\end{tabular}

B. Actual Data Embedding Capacity and the PSNR of a Marked Image

In this way the actual data embedding capacity $\mathrm{C}$ is calculated as follows: 


$$
\mathrm{C} \approx(H(a+1)+H(a+2)-H(b)) \times \frac{2}{3}
$$

The lower bound of the PSNR of a watermarked image is larger than 48db..

\section{Robust Watermarking}

In this section, we will describe how to embed watermark in the remaining part. The detailed steps are described as follows:

1) We split the remaining part of the image into nonoverlapped blocks of $8 \times 8$ and then each block is DCTtransformed. We calculate the value of the ith block:

$$
Q_{i}=\left|f_{i}(0,1)\right|+\left|f_{i}(1,1)\right|+\left|f_{i}(1,0)\right|,
$$

where $f_{i}(0,1) 、 f_{i}(1,1) 、 f_{i}(1,0)$ are three coefficients of low DCT Frequency.

2) Any $Q_{i}$ is qualified to embed $W_{2 i}$ via the following equations:

$$
Q_{i w}=\left\{\begin{array}{ll}
(2 n+1) \Delta & \text { when } W_{2 i}=1 \\
2 n \Delta & \text { when } W_{2 i}=0
\end{array},\right.
$$

where $\Delta$ is the quantization parameter. After quantization the new coefficient is defined as:

$$
\left\{\begin{array}{l}
f_{i w}(0,1)=f_{i}(0,1)+\frac{f_{i}(0,1)\left(Q_{i}{ }^{\prime}-Q_{i}\right)}{\left|f_{i}(0,1)\right|+\left|f_{i}(1,1)\right|+\left|f_{i}(1,0)\right|} \\
f_{i w}(1,1)=f_{i}(1,1)+\frac{f_{i}(1,1)\left(Q_{i}{ }^{\prime}-Q_{i}\right)}{\left|f_{i}(0,1)\right|+\left|f_{i}(1,1)\right|+\left|f_{i}(1,0)\right|} \\
f_{i w}(1,0)=f_{i}(1,0)+\frac{f_{i}(1,0)\left(Q_{i}{ }^{\prime}-Q_{i}\right)}{\left|f_{i}(0,1)\right|+\left|f_{i}(1,1)\right|+\left|f_{i}(1,0)\right|}
\end{array}\right.
$$

3) The IDCT is implemented to produce the watermarked remaining part of the image $I_{1 w}$.

4) $I_{1 w}$ and $I_{0 w}$ forms the image $I_{w}$.

D. The PSNR of a Dual Marked Image and the Quantization Parameter

$$
\begin{gathered}
P S N R=10 \times \log _{10}\left(\frac{255 \times 255}{M S E}\right) \\
M S E=\frac{\sum_{\mathrm{i}=0}^{511} \sum_{\mathrm{j}=0}^{511}\left(\mathrm{I}^{\mathrm{w}}(\mathrm{i}, \mathrm{j})-\mathrm{I}(\mathrm{i}, \mathrm{j})\right)^{2}}{512^{2} * 512}
\end{gathered}
$$

$$
\sum_{i=0}^{511} \sum_{j=0}^{511}\left(I^{w}(i, j)-I(i, j)\right)^{2}=64 \times 64 \times \sum_{i=0}^{7} \sum_{j=0}^{7}\left(I^{w}(i, j)-I(i, j)\right)^{2}
$$

$$
\begin{aligned}
& \sum_{\mathrm{i}=0}^{7} \sum_{\mathrm{j}=0}^{7}\left(\mathrm{I}^{\mathrm{w}}(\mathrm{i}, \mathrm{j})-\mathrm{I}(\mathrm{i}, \mathrm{j})\right)^{2} \\
& =\sum_{\mathrm{j}=0}^{7} \sum_{\mathrm{j}=0}^{7}\left\{a(0) a(1)\left[f^{w}(0,1)-f(0,1)\right] \cos _{j}\right. \\
& +a(1) a(1)\left[f^{w}(1,1)-f(1,1)\right] \cos _{j} \cos _{i} \\
& \left.\quad+a(0) a(1)\left[f^{w}(1,0)-f(1,0)\right] \cos _{i}\right\}^{2}
\end{aligned}
$$

$$
\begin{aligned}
& \text { Where } a(0)=\sqrt{\frac{1}{8}}, a(1)=\sqrt{\frac{2}{8}} \\
& \cos _{j}=\cos \frac{(2 j+1) \pi}{2 \times 8}, \cos _{i}=\cos \frac{(2 i+1) \pi}{2 \times 8} .
\end{aligned}
$$

$$
\begin{aligned}
& \sum_{\mathrm{i}=0}^{7} \sum_{\mathrm{j}=0}^{7}\left(\mathrm{I}^{\mathrm{w}}(\mathrm{i}, \mathrm{j})-\mathrm{I}(\mathrm{i}, \mathrm{j})\right)^{2} \\
& =\sum_{\mathrm{j}=0}^{7} \sum_{\mathrm{j}=0}^{7} \frac{\Delta^{2}}{36}\left\{a(0) a(1) \cos _{j}+a(1) a(1) \cos _{j} \cos _{i}\right. \\
& \left.+a(0) a(1) \cos _{i}\right\}^{2} \\
& \approx 0.10036 \Delta^{2}
\end{aligned}
$$

So we can find

$$
\begin{aligned}
& \text { MSE }=\frac{\sum_{\mathrm{i}=0}^{511} \sum_{\mathrm{j}=0}^{511}\left(\mathrm{I}^{\mathrm{w}}(\mathrm{i}, \mathrm{j})-\mathrm{I}(\mathrm{i}, \mathrm{j})\right)^{2}}{512 * 512} \\
& =\frac{\left(\frac{512 \times 512}{8 \times 8}-\frac{144 \times 120}{8 \times 8}\right) * 0.10036 \Delta^{2}+144 \times 120}{512 * 512}
\end{aligned}
$$

If PSNR $>48, \Delta<25.66$. In our paper we choose 25 .

\section{THE RECOVERY OF ROI AND THE EXTRACTION OF ROBUST WATERMARK}

\section{A. Recovery of ROI and Watermark Extraction}

1) Scan the watermarked image I' in the same sequential order as in embedding steps. Record the pixels with its gray value $a(L), a+1(M), a+2(R)$, the watermark is extracted and the gray value can be calculated through the Template.

2) Scan the image again, . all the pixel gray values (satisfying $\mathrm{a}<\mathrm{x}<\mathrm{b}+1$ ) are decreased by 1 , and the original gray value can be recover.

\section{B. Extraction of Robust Watermark}

We split the corrupted watermarked image $I_{w}{ }^{\prime}$ into non- 
overlapped blocks of $8 \times 8$. Let $f_{\text {iw }}{ }^{\prime}(0,1), f_{i w}{ }^{\prime}(1,1)$ and $f_{i w}{ }^{\prime}(0,1)$ denote the DCT coefficients of the ith block as in embedding. Then we compute $Q_{i w}{ }^{\prime}$ by:

$$
Q_{i w}{ }^{\prime}=\left|f_{i w}{ }^{\prime}(0,1)\right|+\left|f_{i w}{ }^{\prime}(1,1)\right|+\left|f_{i w}{ }^{\prime}(1,0)\right| .
$$

The robust watermark $W_{2}{ }^{\prime}$ is extracted by:

$$
W_{2 i}{ }^{\prime}= \begin{cases}1 & \text { when }\left\lfloor\frac{Q_{i}{ }^{\prime}}{\Delta}+0.5\right\rfloor \text { is odd } \\ 0 & \text { when }\left\lfloor\frac{Q_{i}{ }^{\prime}}{\Delta}+0.5\right\rfloor \text { is even }\end{cases}
$$

\section{A. Reversible Watermark}

The proposed reversible watermarking algorithm has been applied to many different type of images, including some commonly used images, medical images (Figure 3), texture images(Figure 4) and aerial images(Figure 5). The results are presented in Table2.

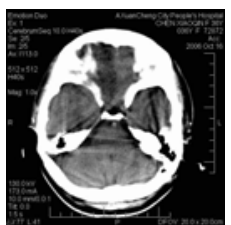

(1)

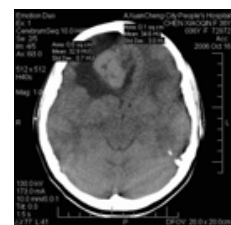

(2)

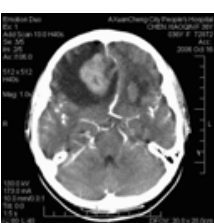

(3)
FIGURE IV. MEDICAL IMAGE 1-3

TABLE II. COMPARISON BETWEEN NI'S SCHEME AND OUR PROPOSED METHOD ON IMAGES TABLE TYPE STYLES

To determine if a watermark exists, we compute the normalized correlation NCr between $W_{2}{ }^{\prime}$ and $W_{2}$ as follows:

$$
N C_{r}=\frac{W_{2 i} \bullet W_{2 i}{ }^{\prime}}{\left(W_{2 i} \bullet W_{2 i}\right)^{\frac{1}{2}}}
$$

\section{EXPERIMENTAL RESULTS}

To evaluate the performance of the proposed watermarking algorithm, a gray Lena image $(512 \times 512$, Figure 1$)$ is the host images, and the face of Lena is the ROI $(120 \times 144)$. The reversible watermark $(40 \times 10)$ and the robust watermark ( 55 $\times 55$ ) are shown in Figure 2. Two dual watermarked images are shown in Figure 3. $\Delta$ is 25 for robust watermark, which can get the good comprise between invisibility and robustness.
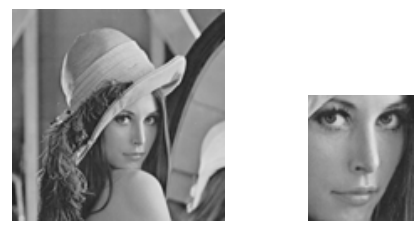

FIGURE I. LENA AND ROI

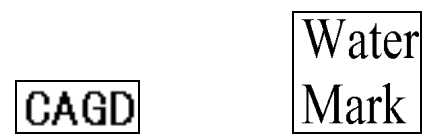

FIGURE II. REVERSIBLE WATERMARK AND ROBUST WATERMARK

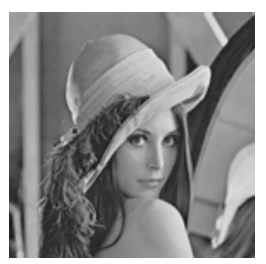

FIGURE III. THE LENA AFTER EMBEDDING MULTIPLE WATERMARKS(PSNR=48.9297)

\begin{tabular}{|l|l|l|l|l|}
\hline \multicolumn{1}{|c|}{$\begin{array}{c}\text { Image } \\
(512 \times 512 \times 8)\end{array}$} & \multicolumn{2}{c|}{ Ni } & \multicolumn{2}{c|}{ our } \\
\cline { 2 - 5 } & payload & PSNR & payload & PSNR \\
\hline Babala & 4563 & 48.1693 & 4760 & 48.3250 \\
\hline Boat & 7301 & 48.3620 & 10462 & 48.2973 \\
\hline Airplane & 16171 & 48.2765 & 17792 & 48.4141 \\
\hline Lena & 5460 & 48.1764 & 5512 & 48.2173 \\
\hline Medical image1 & 56139 & 48.6181 & 74790 & 48.8520 \\
\hline Medical image2 & 50560 & 48.5687 & 67348 & 48.7853 \\
\hline Medical image3 & 50222 & 48.5658 & 66866 & 48.7699 \\
\hline Texture image1 & 15300 & 48.2588 & 15886 & 48.3858 \\
\hline Texture image2 & 17750 & 48.2796 & 19220 & 48.4268 \\
\hline Texture image3 & 22988 & 48.3238 & 23548 & 48.4965 \\
\hline Aerial image 1 & 10824 & 48.2221 & 11292 & 48.3094 \\
\hline Aerial image 2 & 6365 & 48.1838 & 6562 & 48.2365 \\
\hline Aerial image 3 & 10128 & 48.2236 & 10400 & 48.3037 \\
\hline
\end{tabular}

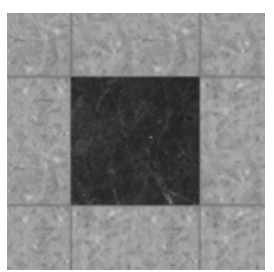

(1)

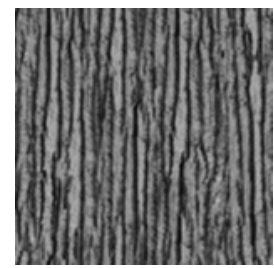

(2)

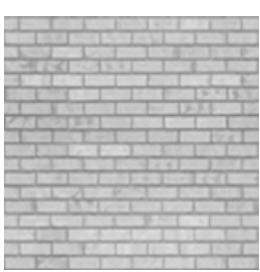

(3)
FIGURE V. TEXTURE IMAGE 1-3

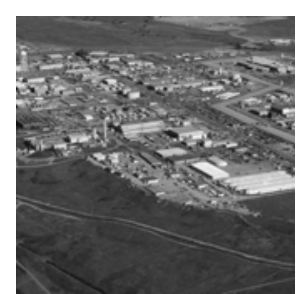

(1)

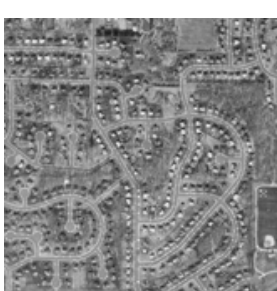

(2)

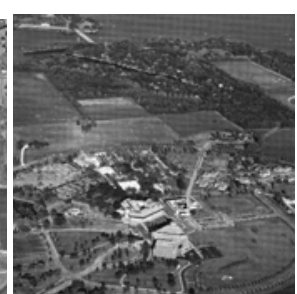

(3)
FIGURE VI. MEDICAL IMAGE 1-3 


\section{B. Robust Watermark}

To check the robustness of the watermark, we perform several attacks on the watermarked image:

1) JPEG;

2) Image processing operation;

3) Geometry attack.

JPEG (JP) with QF (Quality factor) =35\%, 40\%, 45\%, 50\% $60 \%, 70 \%, 80 \%, 90 \%, 100 \%$, The image processing operation includes: $3 \times 3$ median filtering, $5 \times 5$ median filtering, $7 \times 7$ median filtering, $3 \times 3$ Gaussian filtering, $5 \times 5$ Gaussian filtering, jitter attack, gray stretching, blurring, sharpening, changing the brightness/contrast, $\gamma$-emendation, histogram equalization, uniform noise adding, and salt and pepper noise addition. Geometry attack includes: image scaling (SC) with $50 \%$, 110\%, 150\%,200\%, image rotation 0.25,clipping after rotation, slight affine attack. Three kinds of attacks are selected to test the robustness of our watermarking scheme. Figure 6 explains the robust watermark detection results.

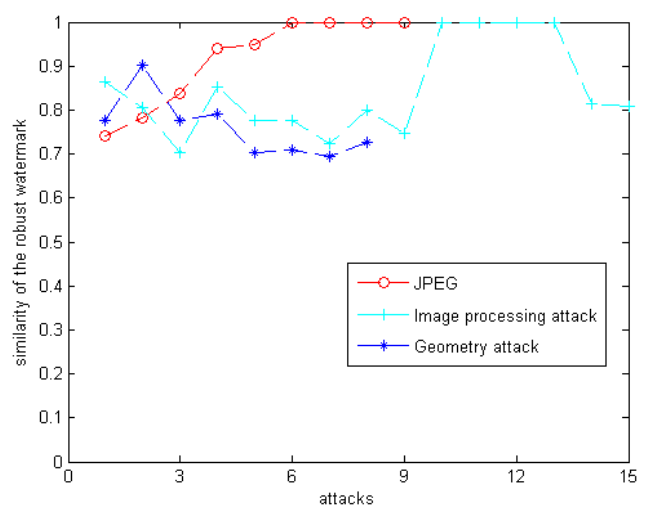

FIGURE VII. THE SIMILARITY OF THE EXTRACTED ROBUST WATERMARK AFTER DIFFERENT ATTACKS

\section{CONCLUSION}

In this paper, we propose a novel multiple watermarking scheme which embeds the reversible watermark in ROI (region of interest) by improving the Ni's algorithm, and the robust watermark in non-ROI by quantizing the low frequency coefficients. The watermarks can simultaneously achieve copyright protection and content authentication, and the ROI of the watermarked image can be recovered completely if it isn't attacked. The experimental results illustrate that the pure payload of the proposed reversible watermarking algorithm is higher 10 percent than Ni's scheme, and the robust watermark can endure common image attacks.

\section{ACKNOWLEDGMENT}

The authors would like to thank the National Natural Science Foundation of China (No. 61302190).

\section{REFERENCES}

[1] Sharkas M., ElShafie D. R., Hamdy N., A Novel Dual-Purpose Image Watermarking Technique. International journal of Signal Processing, 2006, 3(4):252-256.

[2] Shieh C. S., Huang H. C., Wang F.-H., Pan J.-S., An embedding algorithm for multiple watermarks. Journal of Information Science and Engineering 2003, 19(1): 381-395.

[3] Lu C.-S., Liao H.-Y. M., Multipurpose watermarking for image authentication and protection. IEEE Trans. Image Processing, 2001, 10(10):1579-1592.

[4] Honsinger C.W., Jones P., Rabbani M. and J. C. Stoffel, Lossless recovery of an original image containing embedded data, U.S patent application, 1999, Docket No 77 102/E-D.

[5] W. Zhang, K. Ma, N. Yu, Reversibility improved data hiding in encrypted images, Signal Process. 94 (2014) 118-127.

[6] X. Zhang, "Reversible data hiding in encrypted image," IEEE Signal Process. Lett., vol. 18, no. 4, pp. 255-258, Apr. 2011.

[7] X. Zhang, "Separable reversible data hiding in encrypted image," IEEE Trans. Inf. Forensics Security, vol.7, pp.826-832, Apr. 2012.

[8] M.Fujiyoshi, Separable reversible data hiding in encrypted images with histogram permutation, 2013 IEEE International Conference on Information Communication and Embedded Systems (ICICES), ICMEW2013, Page(s):1-4.

[9] Ni Z., Shi Y.Q., Ansari N., Su W., Reversible data hiding. IEEE Transactions on Circuits Systems and Video Technology, 2006, 16(3): 354-362. 